\title{
Logiques d'acteurs et intercommunalité en Franche-Comté
}

Behaviours of actors and intercommunality in Franche-Comté

Verhalten der Fachleute und interkommunale Zusammenarbeit in Franche-

Comté

Jean-Claude Chevailler et Philippe Signoret

\section{(2) OpenEdition}

Journals

Édition électronique

URL : http://journals.openedition.org/rge/1514

DOI : $10.4000 /$ rge.1514

ISSN : 2108-6478

Éditeur

Association des géographes de l'Est

Édition imprimée

Date de publication : 1 juin 2007

ISSN : 0035-3213

Référence électronique

Jean-Claude Chevailler et Philippe Signoret, « Logiques d'acteurs et intercommunalité en FrancheComté », Revue Géographique de l'Est [En ligne], vol. 47 / 3 | 2007, mis en ligne le 04 novembre 2011, consulté le 08 septembre 2020. URL : http://journals.openedition.org/rge/1514 ; DOI : https://doi.org/ $10.4000 /$ rge. 1514

Ce document a été généré automatiquement le 8 septembre 2020

Tous droits réservés 


\title{
Logiques d'acteurs et intercommunalité en Franche- Comté
}

\author{
Behaviours of actors and intercommunality in Franche-Comté \\ Verhalten der Fachleute und interkommunale Zusammenarbeit in Franche- \\ Comté
}

Jean-Claude Chevailler et Philippe Signoret

\section{Introduction}

1 La France se singularise par son organisation administrative du territoire et une dispersion de sa population entre plus de 36500 communes. Ce particularisme s'oppose aux exigences qu'impose la concurrence qui s'exerce désormais entre les territoires, pas seulement à un échelon national. Même si la France connaît, comme tous les pays du continent, le phénomène de métropolisation, il n'en demeure pas moins que la très grande majorité des communes sont de petite, voire de très petite taille. Ce constat soulève une multitude de questions, notamment autour des problèmes d'aménagement et de développement du territoire.

2 La Franche-Comté ne déroge pas à la règle. Avec 1786 communes $\left(4,88 \%^{1}\right.$ des communes françaises de métropole et $3 \%$ de la surface du territoire) et 1158440 habitants (seulement 1,9\% de la population), elle est l'une des plus petites régions de France. Elle présente également une forte dispersion de sa population. La population communale moyenne est de 649 habitants contre 1640 au niveau national et la part des communes de moins de 2000 habitants y est nettement plus importante $(95,3 \%$ des communes contre $87,3 \%$ ). La région apparaît par ailleurs dépourvue d'une véritable armature urbaine, visible aux niveaux national et européen : Besançon, capitale régionale, accueille seulement 122000 habitants et Belfort n'en compte qu'un peu plus de 50000 . 
3 L'analyse départementale permet de dégager trois situations contrastées (cf. tableaux 1 et 2). Le Doubs présente une hiérarchie urbaine légèrement renforcée, tout en restant largement en retrait par rapport à la référence nationale. Besançon réunit en effet moins du quart de la population du département. Le Jura et la Haute-Saône présentent un caractère rural renforcé, avec une très forte proportion de petites communes et l'absence de centre urbain de plus de 50000 habitants. Dans le Jura, le chef-lieu, Lonsle-Saunier, est moins peuplé que Dole. Le Territoire de Belfort se rapprocherait des références nationales mais en proposant un espace plus restreint (102 communes contre plus de 500 dans les autres départements) et un chef-lieu qui représente plus du tiers de la population.

Ainsi, près d'un Franc-Comtois sur deux (46,7\% contre $25,3 \%$ au plan national) vit dans une commune de moins de 2000 habitants et le phénomène est davantage marqué dans le Jura et la Haute-Saône (respectivement 58,1 \% et 62,4 \% de la population) que dans le Doubs ou le Territoire de Belfort (respectivement $37,8 \%$ et 30,9\%).

Dans ces conditions générales, on comprend la nécessité, ici peut-être plus qu'ailleurs, de favoriser les rapprochements entre communes pour qu'elles puissent mettre en oeuvre les politiques souhaitées par leurs populations et réduire, dans la mesure du possible, une déprise démographique qui menace une partie importante du territoire comtois. Or, en l'absence de rapprochements spontanés, cela ne peut s'opérer que par les adaptations de la législation nationale.

Depuis plus d'un siècle, avec la création des premiers syndicats, les communes ont été incitées à se rapprocher pour regrouper leurs moyens et faire face à des défis comme l'électrification, l'adduction d'eau, l'assainissement... De nouvelles préoccupations liées à la relative désertification des campagnes et à l'évolution de la société (transports, équipements scolaires et périscolaires, équipements culturels ou sportifs, action sanitaire et sociale, etc.) sont ensuite venues alimenter les réflexions des acteurs du territoire. Ainsi, les regroupements se sont poursuivis tout d'abord avec les Communautés urbaines ${ }^{2}$ qui associent plusieurs communes d'un seul tenant et sans enclave formant un ensemble de plus de 500000 habitants. Dans la période récente, le mouvement s'est amplifié avec la création de nouveaux Établissements publics de coopération intercommunale (EPCI) permettant de prendre en compte la nature des communes et le caractère polarisé ou non autour d'un centre : les Communautés de communes $^{3}$ et les Communautés d'agglomération ${ }^{4}$.

7 Parallèlement, le législateur a créé en deux étapes, sous le vocable de Pays ${ }^{5}$, des territoires de projets. Il a remplacé le Schéma directeur d'aménagement et d'urbanisme par un instrument de planification : le Schéma de cohérence territoriale (SCOT) ${ }^{6}$. Les problématiques générales ont évolué. Il s'agit désormais de lutter contre l'étalement urbain, synonyme de consommation d'espace, de coûts supplémentaires en termes d'équipements pour les collectivités locales et de nuisances (encombrements, pollution) occasionnées par des déplacements toujours plus nombreux (Chevailler, 2005).

Comment ne pas se perdre dans les arcanes administratifs français si l'on considère les échelons départemental, régional, national et européen ? Peut-on éviter le scepticisme qui envahit toute personne à qui l'on essaie d'expliquer le fonctionnement de nos institutions?

9 Nous tentons de dresser une photographie précise de l'intercommunalité afin d'en évoquer les incertitudes, les insuffisances et de mettre en perspective d'éventuelles 
améliorations à apporter. Qu'en est-il de la dynamique intercommunale ? La FrancheComté parviendra-t-elle à compenser l'absence d'armature urbaine par une intercommunalité active pour conserver une place sur l'échiquier international?

Tableau 1 : Distribution des communes selon leur taille (\%)

\begin{tabular}{|l|c|c|c|c|c|c|}
\hline \multicolumn{1}{|c|}{ Territoire concerné } & $\begin{array}{c}\text { Nombre total } \\
\text { de communes }\end{array}$ & $<2000$ & $\begin{array}{r}2000- \\
9999\end{array}$ & $\begin{array}{c}10000- \\
49999\end{array}$ & $\begin{array}{c}50000- \\
199999\end{array}$ & $\geq 200000$ \\
\hline Doubs & 594 & 93,6 & 5,6 & 0,7 & 0,2 & 0,0 \\
Jura & 545 & 97,1 & 2,4 & 0,6 & 0,0 & 0,0 \\
Haute-Saône & 545 & 96,9 & 2,8 & 0,4 & 0,0 & 0,0 \\
Territoire de Belfort & 102 & 87,3 & 11,8 & 0,0 & 1,0 & 0,0 \\
Franche-Comté & 1786 & 95,3 & 4,1 & 0,5 & 0,1 & 0,0 \\
France Métropolitaine & 36565 & 87,3 & 10,3 & 2,1 & 0,3 & 0,027 \\
\hline
\end{tabular}

Source : INSEE - RGP 99

Tableau 2 : Distribution de la population légale selon la taille des communes (\%)

\begin{tabular}{|l|c|c|c|c|c|c|}
\hline \multicolumn{1}{|c|}{ Territoire concerné } & $\begin{array}{c}\text { Population } \\
\text { totale }\end{array}$ & $<2000$ & $\begin{array}{c}2000- \\
9999\end{array}$ & $\begin{array}{c}10000- \\
49999\end{array}$ & $\begin{array}{c}50000- \\
199999\end{array}$ & $\geq 200000$ \\
\hline Doubs & 515319 & 37,8 & 23,6 & 14,9 & 23,7 & 0,0 \\
Jura & 261922 & 58,1 & 19,4 & 22,4 & 0,0 & 0,0 \\
Haute-Saône & 239101 & 62,4 & 25,3 & 12,3 & 0,0 & 0,0 \\
Territoire de Belfort & 142098 & 30,9 & 32,1 & 0,0 & 37,0 & 0,0 \\
Franche-Comté & 1158440 & 46,7 & 24,0 & 14,2 & 15,1 & 0,0 \\
France Métropolitaine & 59951435 & 25,3 & 25,5 & 26,0 & 14,4 & 8,9 \\
\hline
\end{tabular}

Source : INSEE - RGP 99

10 Pour les besoins de l'étude, nous nous sommes appuyés sur les Schémas départementaux d'orientation de l'intercommunalité (SOI, 2007), commandés aux Préfets par le Ministre délégué aux Collectivités territoriales, validés par les Commissions départementales de coopération intercommunale et par les premières synthèses réalisées (ADCF 2007, Le Répertoire administratif 2007).

\section{L'intercommunalité en Franche-Comté}

11 Au tournant du troisième millénaire, on comptait dans le pays près de 15000 Syndicats intercommunaux à vocation unique (SIVU) et 1700 Syndicats intercommunaux à vocation multiples (SIVOM). On doit leur ajouter les syndicats mixtes, également créés en 1955, qui réunissent au sein d'une même structure des partenaires de natures différentes (Région, département, EPCI, communes, centre intercommunal d'action sociale, etc.). Tous ces syndicats sont des EPCI sans fiscalité propre dont les ressources sont constituées exclusivement par les contributions des membres les composant.

En Franche-Comté, on comptait, en 2006, si l'on exclut les syndicats mixtes, pas moins de 692 EPCI sans fiscalité propre (cf. tableau 3) dont un tiers dans le Doubs, un quart dans le Jura, un tiers en Haute-Saône et le restant dans le Territoire de Belfort. 

Saône et du Territoire de Belfort. Toutefois, en termes de dynamique, il semblerait que ce soit dans le Jura que l'on observe les plus gros efforts de regroupement avec une moyenne de 3,1 communes par syndicat. l'eau, assainissement, collecte des ordures ménagères, politique scolaire et périscolaire constituent la justification essentielle des coopérations intercommunales.

Tableau 3 : Les syndicats de coopération intercommunale début 2006

\begin{tabular}{|l|r|c|c|c|c|}
\hline & $\begin{array}{c}\text { Population } \\
\text { (RGP 1999) }\end{array}$ & Communes & Syndicats & Pop/synd & Comm./synd \\
\hline Doubs & 499062 & 594 & 230 & 2170 & 2,6 \\
Jura & 250857 & 545 & 177 & 1417 & 3,1 \\
Haute-Saône & 229732 & 545 & 231 & 995 & 2,4 \\
Territoire de Belfort & 137408 & 102 & 54 & 2545 & 1,9 \\
Franche-Comté & 1117059 & 1786 & 692 & 1614 & 2,6 \\
\hline
\end{tabular}

Source : Préfectures

Tableau 4 : Répartition des communes au 1/1/2007 selon les EPCI à fiscalité propre

\begin{tabular}{|l|c|c|r|r|r|r|r|r|r|}
\hline & $\begin{array}{c}\text { Nb total } \\
\text { de } \\
\text { communes }\end{array}$ & $C A$ & $\begin{array}{c}\text { Nb de } \\
\text { communes }\end{array}$ & $C C$ & $\begin{array}{c}\text { Nb de } \\
\text { communes }\end{array}$ & $\begin{array}{c}\text { Total } \\
E P C l\end{array}$ & $\begin{array}{c}\text { Total } \\
\text { communes }\end{array}$ & $\%$ & $\begin{array}{c}\text { Communes } \\
\text { orphelines }\end{array}$ \\
\hline Doubs & 594 & 2 & 87 & 28 & 474 & 30 & 561 & 94,4 & 33 \\
Jura & 545 & 0 & 0 & 31 & 524 & 31 & 524 & 96,1 & 21 \\
Haute-Saône & 545 & 0 & 0 & 30 & 466 & 30 & 466 & 85,5 & 79 \\
Territoire de Belfort & 102 & 1 & 30 & 5 & 67 & 6 & 97 & 95,1 & 5 \\
Franche-Comté & 1786 & 3 & 117 & 94 & 1531 & 97 & 1648 & 92,3 & 138 \\
\hline
\end{tabular}

Source : DGCL

Mais derrière les chiffres se cache une réalité que les informations recueillies ne permettent pas de décrire en toute rigueur et nombre de questions restent sans réponse. Le territoire est-il totalement couvert par des EPCI sans fiscalité propre ? Où se situent les zones blanches? Comment se répartissent les transferts de compétences ? Quelle est la part de la superposition des périmètres ? La logique spatiale est-elle vraiment prise en compte?

Les EPCI à fiscalité propre, plus récents, devraient apporter des précisions à toutes ces questions. C'est pourquoi nous nous intéresserons particulièrement aux communautés de communes (CC) et aux communautés d'agglomération (CA) de Franche-Comté ; il n'y a pas de Communauté Urbaine en pays comtois.

Les districts, dont les plus anciens ont été créés au début des années soixante, ont peu à peu disparu et se sont transformés, soit en CC, soit en CA, qui regroupent des communes géographiquement contiguës, ce qui exclut, à la différence des syndicats, toute possibilité d'enclave. Les CA doivent répondre aux mêmes exigences quant à la définition de leur périmètre et compter en outre, lorsqu'il ne s'agit pas du chef-lieu 
départemental, au moins 50000 habitants autour d'une ou de plusieurs communes centres d'au moins 15000 habitants.

18 L'organisation du territoire national en EPCI à fiscalité propre est un réel succès : début 2007, on compte, selon la Direction générale des collectivités locales (DGCL), 2400 CC et 169 CA.

\section{A. La dynamique intercommunale comtoise}

En Franche-Comté, 43 \% des EPCI à fiscalité propre ont été créés à partir de 1999 et les plus anciennes s'appuyaient le plus souvent sur un syndicat intercommunal ou un district. Le passage du district à la CA se fit de façon naturelle à Belfort et à Montbéliard ; avec un district urbain de Belfort créé en 1973 et celui du Pays de Montbéliard dès 1959, l'intercommunalité était déjà bien installée.

Les discussions furent plus serrées dans la capitale régionale, où l'intercommunalité était plus récente (district créé en 1995). On assista, lors de la transformation, à un jeu d'acteurs qui opposa l'ensemble des élus bisontins à ceux des élus de la périphérie qui soutenaient la position du Président du Conseil Général (Chevailler, 2003). L'âpreté du débat tenait moins aux oppositions politiciennes, qui ont certes joué leur rôle, qu'à la structure particulière de la future CA. En effet, à la ville centre de 120000 habitants venaient s'ajouter pas moins de 56 communes ; deux communes supplémentaires rejoindront plus tard la CA, ce qui en fait une des plus grandes de France du point de vue du nombre de communes. La plus grande de ces communes ne compte guère plus de 3000 habitants et la grande distorsion de taille entre le centre et les autres communes de la CA explique en grande partie les craintes des habitants portées par certains maires de la périphérie bisontine. 
Figure 1 : L'intercommunalité en Franche-Comté en 1999 et 2007

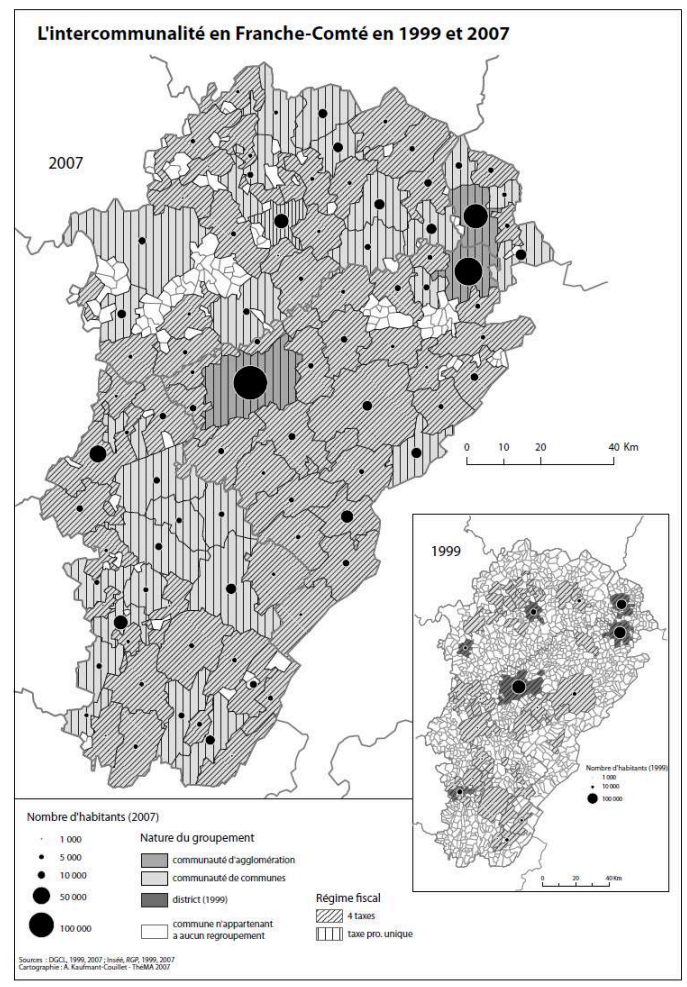

\section{B. Un système de gouvernance comparable et un régime fiscal qui fait la différence}

Les CC et les CA sont administrées par un conseil communautaire dont les membres sont désignés au second degré par les conseils municipaux. Si, en milieu rural, la répartition des sièges entre les communes en fonction du nombre d'habitants ne pose pas de problème, le poids important de la ville centre ( $70 \%$ de la population) dans la CA du Grand Besançon imposa une approche plus modérée. Les élus bisontins acceptèrent que Besançon soit minoritaire au conseil communautaire au profit d'un débat renforcé avec les communes périphériques.

Les CC et les CA disposent d'un budget alimenté par une fiscalité propre complétée d'aides diverses et de recettes propres (produits de taxes, redevances ou contributions correspondant à des services assurés par elles).

Les CC peuvent opter, soit pour une fiscalité propre additionnelle qui se surajoute à celle des communes qui continuent de percevoir leur fiscalité sur les quatre taxes directes (taxes foncières, taxe d'habitation, taxe professionnelle), soit pour une fiscalité propre fondée sur une Taxe professionnelle unique (TPU). Les CA disposent quant à elles d'une fiscalité composée essentiellement, et souvent exclusivement, de la seule TPU.

Qu'il s'agisse d'une CC ou d'une CA, et sans entrer dans le détail, le budget de l'EPCI à TPU est toutefois diminué d'une attribution de compensation reversée aux communes membres et de dotations de solidarité communautaire qui contribuent à maintenir un lien entre l'EPCI et ses membres. 

membres dans les groupes de compétences obligatoires (deux pour les $\mathrm{CC}$ et six pour les $\mathrm{CA}$ ) et optionnelles (au moins une pour les CC et au moins trois pour les CA). Les CA ont ici un rôle accru dans l'organisation et la gestion du territoire mais aussi en matière d'habitat et de développement social. À souligner également qu'une commune ou un syndicat intercommunal ne peut plus exercer individuellement une compétence transférée à un EPCI. problématiques liées à l'environnement et au cadre de vie font l'objet d'un intérêt particulier ( $68 \%$ des EPCI), suivies de près par les équipements (54,6 \%). Les questions de voiries sont également prégnantes en Franche-Comté, si ce n'est dans le Doubs où les questions d'accessibilité sont peut-être moins prononcées.

\section{Une taille des EPCI souvent modeste}

La taille des EPCI est un élément très important, si l'on considère que l'aménagement de l'espace et le développement économique, pour ne reprendre que les compétences obligatoires, exigent une taille suffisante pour avoir une signification véritable, en particulier du point de vue de l'offre de services, qu'ils soient publics ou privés.

Bien sûr, les 3 CA (Besançon, Montbéliard et Belfort) dépassent les 50000 habitants. Mais, on notera que pas moins de $14 \mathrm{CC}$ comptent moins de 2500 habitants, ce qui, on le verra, ne laisse pas indifférents les représentants de l'État. 
Tableau 5 : Compétences des $\mathrm{CC}$ et des $\mathrm{CA}$

\begin{tabular}{|l|l|c|c|}
\hline \multirow{5}{*}{} & \multicolumn{1}{|c|}{ compétences } & CC & $C A$ \\
\hline \multirow{5}{*}{ Obligatoires } & Aménagement de l'espace & $\checkmark$ & $\checkmark$ \\
\cline { 2 - 4 } & $\begin{array}{l}\text { Actions de développement économique intéressant l'ensemble } \\
\text { de la communauté }\end{array}$ & $\checkmark$ & $\checkmark$ \\
\hline & Schémas directeur et de secteur & & $\checkmark$ \\
\hline & Habitat & & $\checkmark$ \\
\hline & Développement urbain, local et insertion économique et sociale & & $\checkmark$ \\
\hline & Prévention de la délinquance & & $\checkmark$ \\
\hline \multirow{5}{*}{ Optionnelles } & Protection et mise en valeur de l'environnement & $\checkmark$ & 0 bligatoire \\
\hline & Politique du logement et cadre de vie & $\checkmark$ & $\checkmark$ \\
\hline & Création, aménagement et entretien de la voirie & $\checkmark$ & $\checkmark$ \\
\hline & Equipements culturels et sportifs d'intérêt communautaire & & $\checkmark$ \\
\hline & Assainissement & & $\checkmark$ \\
\hline & Eau & & $\checkmark$ \\
\hline
\end{tabular}

Tableau 6 : La prise de compétence par les EPCI franc-comtoises

\begin{tabular}{|l|c|c|c|c|c|}
\hline \multicolumn{1}{|c|}{ en \% } & Doubs & Jura & Haute-Saone & $\begin{array}{c}\text { Territoire } \\
\text { de Belfort }\end{array}$ & $\begin{array}{c}\text { Franche- } \\
\text { Comté }\end{array}$ \\
\hline Développement économique & 100 & 100 & 100 & 100 & 100 \\
Aménagement de l'espace & 100 & 100 & 100 & 100 & 100 \\
Equilibre social de I'habitat & 13,3 & 3,2 & 16,7 & 33,3 & 12,4 \\
Politique de la ville & 6,7 & 0,0 & 0,0 & 16,7 & 3,1 \\
Transport & 3,3 & 0,0 & 10,0 & 0,0 & 4,1 \\
Voirie & 16,7 & 41,9 & 70,0 & 83,3 & 45,4 \\
Eau et/ou assainissement & 6,7 & 9,7 & 3,3 & 66,7 & 10,3 \\
Gestion des services d'intérét coll. & 6,7 & 6,5 & 3,3 & 0,0 & 5,2 \\
Environnement et cadre de vie & 63,3 & 61,3 & 76,7 & 83,3 & 68,0 \\
Equipements culturels, sportifs, etc. & 56,7 & 48,4 & 60,0 & 50,0 & 54,6 \\
\hline
\end{tabular}

Source : ADCF

Tableau 7 : Répartition des EPCI à fiscalité propre selon la taille

\begin{tabular}{|l|c|c|c|c|c|c|c|}
\hline & $<2500$ & $\begin{array}{r}2500- \\
5000\end{array}$ & $\begin{array}{c}5000- \\
10000\end{array}$ & $\begin{array}{r}10000- \\
20000\end{array}$ & $\begin{array}{r}20000 \\
50000\end{array}$ & $>50000$ & Total \\
\hline Doubs & 4 & 5 & 14 & 4 & 1 & 2 & 30 \\
Jura & 5 & 7 & 13 & 4 & 2 & 0 & 31 \\
Haute-Saône & 5 & 12 & 6 & 6 & 1 & 0 & 30 \\
Territoire de Belfort & 0 & 2 & 2 & 0 & 1 & 1 & 6 \\
Franche-Comté & 14 & 26 & 35 & 14 & 5 & 3 & 97 \\
\hline
\end{tabular}

Source : DGCL

31 En Franche-Comté, avec $8 \%$ de communes orphelines, des zones blanches persistent ; pour autant, le phénomène est moins marqué qu'au niveau national (10\%). Le Jura (4 $\%)$, le Territoire de Belfort (5\%) et le Doubs (6\%) contribuent à la relative faiblesse de cette proportion régionale. En revanche, en Haute-Saône, le pourcentage de communes qui ne sont pas rattachées à un EPCI s'élève à $14 \%$. Le bon pourcentage régional témoigne de la volonté de ne pas ajouter de nouveaux handicaps qui ferait perdre en 
efficacité les mesures prises notamment en matière d'aménagement du territoire, de développement économique et d'environnement.

\section{E. Des EPCI définis par défaut témoignent des tensions locales}

32 La CAGB englobe 59 communes. Elle a laissé, sur ses marges incluses dans le périmètre du SCOT, une bande (troisième couronne de Besançon) s'arrêtant aux limites du département. Dans cet espace subcontinu, le reliquat de communes de trois cantons - la plupart étaient déjà rattachées à la CAGB - se sont regroupées, constituant ainsi trois CC par défaut. Ceci ne va pas sans soulever ou révéler des tensions entre les acteurs d'un territoire contrasté au sein duquel les pratiques collaboratives et les perspectives de développement peuvent être concurrentielles voire contradictoires.

\section{Les interrogations relatives à la coopération intercommunale}

La synthèse des Schémas d'Orientation de l'Intercommunalité a été réalisée par les services du Ministre délégué aux collectivités territoriales (Intercommunalité, 2007), cela en vue de la réforme que devrait proposer le gouvernement mis en place en mai 2007. La situation franc-comtoise n'est pas sans rappeler les difficultés rencontrées au plan national. Les acteurs locaux peuvent donc sans ambages s'inspirer des préconisations du Ministère.

\section{A. Vers une plus forte rationalisation des périmètres}

Selon la plupart des auteurs, la définition de projets structurants exige une population minimale comprise entre 3500 et 5000 habitants. En deçà de cette taille, il est difficile de réaliser l'efficacité budgétaire nécessaire à l'élaboration et à la mise en oeuvre de projets significatifs. C'est la question de la taille critique des EPCI qui est donc clairement posée.

En milieu rural, l'obtention de la taille critique par le regroupement d'un grand nombre de petites communes peut aller à l'encontre de l'homogénéité nécessaire à l'apparition et au portage de projets communs ; les nombreux syndicats préexistants venant se superposer au territoire d'une $\mathrm{CC}$ contribuent alors au manque de visibilité. Or, les élus manifestent en général le souhait de conserver ces derniers lorsqu'ils ont fait la preuve de leur utilité.

36 À l'autre extrémité de l'échelle, et en milieu (r)urbain, la CC du Pays Lédonien (33 178 habitants) et la CC de l'Agglomération de Vesoul (35 078 habitants) devraient, pour prétendre au statut de CA, gagner respectivement 17000 et 15000 habitants. Cela ne pourrait se faire qu'en intégrant plusieurs dizaines de communes supplémentaires, créant ainsi un territoire dont l'étendue poserait des difficultés en matière de gouvernance et de proximité de l'intervention publique. 


\section{B. Une rationalisation en action}

Incontestablement, le Territoire de Belfort est, du point de vue de la rationalisation des périmètres, le département qui pose le moins de problème. La plus petite des cinq $\mathrm{CC}$ compte 4154 habitants. Cinq communes seulement regroupant 238 habitants, demeurent isolées. Les voeux du Préfet, repris dans le schéma d'orientation de l'intercommunalité, sont de rattacher ces dernières aux EPCI existants. Ainsi, trois des cinq communes isolées rejoindraient la CA Belfortaine, les deux autres la CC du Sud Territoire qui, avec 20653 habitants, est déjà la CC du département la plus peuplée. Le Préfet souhaite également fusionner les quatre autres $\mathrm{CC}$ deux à deux, ce qui permettrait la couverture complète du département par seulement quatre EPCI : une CA et trois CC.

Dans le Doubs, la situation est également satisfaisante du point de vue territorial, puisque seules $4 \mathrm{CC}$ comptent moins de 3000 habitants et que toutes les communes isolées appartiennent à l'arrondissement de Montbéliard ce qui facilitera certainement leur intégration au sein d'une EPCI.

\section{Un émiettement qui vient s'ajouter à un caractère rural}

En Haute-Saône, l'émiettement communal dans ce département au caractère rural marqué reste important. Un grand nombre de communes (79) de petite taille demeurent isolées et les $\mathrm{CC}$ existantes sont elles mêmes de taille réduite : cinq comptent moins de 2000 habitants et douze entre 2000 et 5000 habitants. En 2005, dans une première ébauche de schéma d'orientation, il avait été prévu trois fusions et le rattachement des communes isolées de façon à couvrir l'ensemble du territoire départemental par des EPCI de tailles respectables.

Les regroupements devraient être favorisés par la loi du 23 février 2005, qui prévoit qu'une commune isolée appartenant à une zone de revitalisation rurale ne pourrait plus bénéficier des aides prévues à partir de 2008. Quarante-neuf communes isolées sont concernées et devraient, sous l'influence de cette mesure, intégrer prochainement un EPCI.

41 Après de nombreuses réunions de concertation, l'élaboration du schéma a donné lieu à un projet qui envisage plusieurs possibilités :

- rattachement de communes à une $\mathrm{CC}$ existante : par exemple, à la CC des Quatre Vallées, qui ne compte que trois communes pour 704 habitants, est proposé le rattachement de onze communes isolées qui donnerait une nouvelle structure de 3839 habitants;

- fusion simple : par exemple, fusion de la CC du Pays de Montbozon (21C, $4287 \mathrm{hb}$ ) avec celle du Chanois (6C et $1495 \mathrm{hb}$ );

- redéfinition de la carte intercommunale autour du chef-lieu de département : rattachement de cinq communes à la CC de l'agglomération de Vesoul (l'ensemble passerait à 20 communes et 33439 habitants); fusion de trois CC (Saône Jolie, Agir ensemble, Six villages) et rattachement de trois communes isolées.

Autour de Vesoul, les CC Saône Jolie et Agir ensemble refusent la fusion. La CC des Six Villages serait éclatée, cinq communes demandant le rattachement à la CC de l'agglomération de Vesoul, la sixième à la CC de la Saône Jolie, à laquelle viendraient par ailleurs se rattacher trois autres communes. On constate ainsi que les 
préconisations du schéma d'orientation se heurtent aux voeux des communes et de leur maire soucieux de leur indépendance.

Dans ce contexte, quel degré de latitude sera réservé aux communes? Dans quelle mesure l'autorité centrale sera-t-elle conduite à imposer, en cas d'échec, les solutions les plus conformes à la mise en oeuvre de projets d'aménagements et d'équipements structurants?

\section{Des regroupements imposés}

Le Jura comprenait 32 CC et 21 communes isolées. Le schéma départemental de 1995 prévoyait de ramener le nombre de CC à 21 en regroupant les CC n'atteignant pas une taille suffisante évaluée à 4000 habitants. Il était alors envisagé de fusionner les CC AinAngillon et Malvaux d'une part, les CC du Jura Dolois et du Jura entre Serre et Chaux d'autre part.

Le schéma proposait également trois regroupements souhaitables de deux $\mathrm{CC}$, un autre de trois CC et le rattachement de la CC de Nozeroy au regroupement Ain-Angillon et Malvaux évoqué plus haut. À chaque fois, le regroupement était justifié par la nature des projets qui pouvaient voir le jour dans une intercommunalité rénovée.

Malgré des réticences, et après consultation des instances intéressées, la fusion entre les CC d'Ain- Angillon (26 communes et 16471 habitants) et de Malvaux (6 communes et 1920 habitants) a été autorisée par arrêté préfectoral fin 2006. Afin de supprimer toute enclave, ont également été rattachées à la nouvelle CC six communes isolées, dont 4 contre leur volonté. La nouvelle CC regroupe ainsi 38 communes et 19515 habitants. Le rattachement envisagé de la CC de Nozeroy aurait fait coïncider la CC et le Pays de la Haute-Vallée de l'Ain.

Le Jura fournit ainsi une belle illustration de regroupements imposés par l'autorité préfectorale.

\section{La clarification des compétences}

Outre la rationalisation des périmètres, l'Etat est aujourd'hui très vigilant sur la clarification des compétences entre communes et EPCI. Il s'agit d'éviter les EPCI d'aubaine « coquilles vides » créés pour capter les dotations compensatoires de l'État, et l'enchevêtrement des compétences entre communes et communautés.

Ainsi, le législateur a fixé un délai aux communautés afin qu'elles définissent l'intérêt communautaire pour les compétences soumises à cette définition. En effet, contrairement aux collectivités territoriales qui disposent d'une compétence générale sur leurs territoires, l'EPCI ne dispose que de compétences d'attribution librement transférées par les communes. Dans ce cadre, les services de l'État, par le contrôle de légalité, veillent scrupuleusement au respect des principes de spécialité et d'exclusivité. En vertu du principe de spécialité, l'EPCI ne peut intervenir que dans le champ des compétences qui lui ont été transférées (spécialité fonctionnelle) et à l'intérieur de son périmètre (spécialité territoriale).

50 En vertu du principe d'exclusivité, les EPCI sont les seuls à pouvoir agir dans les domaines se rattachant aux compétences qui leur ont été transférées : les communes ne peuvent plus intervenir dans ces domaines. 
51 Toujours dans le but de clarifier les compétences des EPCI, le législateur a fixé des règles qui obligent les communes à transférer des blocs de compétence, sans scission possible. Ainsi, par exemple, dans le domaine de la voirie, l'investissement et le fonctionnement doivent être exercés par la même personne publique.

52 L'enchevêtrement des compétences était particulièrement patent dans le domaine de l'élimination et de la valorisation des déchets ménagers qui se décomposent en deux sous compétences, la collecte et le traitement. La loi permet à une commune de transférer soit la compétence traitement, soit les deux compétences collecte et traitement à un EPCI. En revanche, la loi interdit à une commune de transférer sa compétence collecte à un EPCI et sa compétence traitement à un autre EPCI (transfert dit « en étoile »). Au sein de la CAGB, mis à part la Ville de Besançon, la plupart des communes étaient dans cette situation en 2005 : collecte confiée à un syndicat et traitement à la CAGB qui l'avait elle-même transféré au Syndicat mixte de Besançon et de sa Région pour le Traitement des déchets (SYBERT).

53 Afin de se mettre en conformité avec la loi, les communes de la CAGB ont décidé de se retirer des syndicats auxquels elles adhéraient pour la compétence collecte, et de la confier à la CAGB à compter du 1er janvier 2006. Ce transfert s'est traduit par la dissolution de quatre syndicats et du transfert des moyens humains et matériels nécessaires à l'exercice de la compétence collecte par la CAGB. Cette réorganisation a également eu pour conséquence l'extension du périmètre du SYBERT qui réunit désormais la CAGB et sept CC, soit 198 communes, représentant un peu plus de 220000 habitants. Il s'agit là d'un exemple de rationalisation importante des moyens, qui plus est dans un domaine qui va prendre une importance grandissante dans un avenir proche (Chevailler, 2007).

54 Néanmoins, avec la loi du 13 août 2004, le législateur a assoupli la portée des principes de spécialité et d'exclusivité. Ainsi, aujourd'hui, la loi autorise les CA à attribuer des subventions à leurs communes membres, et inversement, afin de financer la réalisation ou le fonctionnement d'un équipement, sans détenir la compétence. Mais si le législateur a assoupli la portée de ces principes entre communes et communautés, ce n'est pas encore le cas entre syndicats de communes et communautés.

55 Ainsi, la Préfecture du Doubs a demandé au Tribunal Administratif de Besançon d'annuler la subvention de la CAGB au syndicat intercommunal Roche-Novillars pour la construction d'un gymnase. Néanmoins, par courrier, les services de l'État proposèrent une solution qui consistait en « la dissolution du syndicat, qui aurait pour effet de réintégrer les deux communes membres dans leurs compétences, et donc permettre de bénéficier du fonds de concours de la CAGB ». Une fois la subvention de la CAGB versée, le syndicat pourrait alors être recréé... Ce cas de figure n'est pas unique. Auparavant, dans la même $\mathrm{CAGB}$, le problème s'était posé pour la participation de la $\mathrm{CA}$ à un projet de déplacement d'un stade en raison de sa proximité avec une installation classée SEVESO. Dans ce cas, l'État, qui était à l'origine de la demande de déplacement, s'est montré plus conciliant.

56 À la lumière de ces illustrations, il conviendrait certainement d'envisager un assouplissement de la loi, afin d'éviter la mise en oeuvre de solutions kafkaïennes. 


\section{Intercommunalité et aménagement du territoire}

57 Au nord du département du Jura, le Pays Dolois se compose de six CC : Jura Dolois, Jura entre Serre et Chaux, Plaine Jurassienne, Nord-Ouest Dolois, Val d'Amour et Nord Jura. Cette dernière est contiguë à la $\mathrm{CC}$ doubiste du Val Saint-Vitois qui appartient au SCOT du Grand Besançon. Lorsque le Pays a envisagé de prendre la compétence SCOT, les élus de la CC du Nord-Jura ont refusé d'adhérer arguant du fait que les questions qui les concernaient étaient davantage orientées vers Besançon que vers Dole. Or, la dynamique territoriale en jeu au niveau régional, voire interrégional, rapproche la Région de Dole et le Grand Besançon, alors que le chef-lieu, Lons-le-Saunier, se trouve plutôt dans la zone d'influence de Lyon. Ainsi, à l'initiative des Jurassiens, les élus du SCOT du Grand Besançon et ceux du SCOT de la Région de Dole se sont rapprochés pour évoquer les grands projets qui structurent le territoire : construction de la branche sud de la LGV Rhin- Rhône, amélioration des infrastructures routières, développement d'un aéroport interrégional à Dole- Tavaux, ou adhésion au réseau métropolitain RhinRhône. On se retrouve donc dans le cas paradoxal où la $\mathrm{CC}$ qui se sentait la plus concernée se retrouve en dehors des rencontres et des réflexions communes. Cet exemple, si l'on fait abstraction du jeu des acteurs politiques qui n'est jamais négligeable, montre à quel point l'importance des projets envisagés modifie l'échelle des espaces de planification et de mise en oeuvre. Il est bien évident que ce type de question se retrouve sur l'ensemble du territoire régional.

La concurrence qui s'exerce entre les communes se traduit par un éparpillement des zones d'activités, une dégradation du paysage, voire un affaiblissement de l'efficacité économique. L'instauration d'une TPU, en mettant fin à une concurrence stérile à l'échelon local, constitue le moyen de rationaliser et d'organiser les territoires. $\mathrm{Si}$, pour les CA, elle est une obligation, il n'en est pas de même pour les CC. Il y a donc encore une marge de progression importante, particulièrement, comme on l'a vu, dans le département du Doubs, le Schéma départemental d'orientation mentionnant justement la nécessité d'aller en ce sens.

\section{Conclusion}

Nous avons mis l'accent sur l'évolution de l'intercommunalité en référence à l'aspect territorial, c'est-àdire en rapprochant les compétences du cadre territorial dans lesquelles elles sont exercées au mieux de l'intérêt des populations. Or, dans le contexte global d'un endettement important du pays, il est de bon ton de mettre en lumière un autre aspect de l'intercommunalité : le manque d'efficacité financière.

Les nouvelles structures mises en place viendraient se surajouter aux communes, sans que les économies d'échelles que l'on pourrait en attendre soient évidentes. De nombreux rapports (Mariton 2005, Richard 2006, Dallier 2007) pointent le doigt sur l'accroissement des effectifs communaux qui se poursuit malgré la mise en place de l'intercommunalité, sur l'insuffisance des moyens transférés lors des transferts de compétences ou sur les doublons d'effectifs.

61 Même si elles paraissent parfois excessives, les critiques émises ne sont pas dénuées de tout fondement. En effet, l'exemple de Mulhouse où les services de la ville et de la CA sont fusionnés depuis 2003, bien avant la loi du 13 août 2004 relative aux libertés et 
responsabilités locales, montre que la mutualisation des ressources humaines et financières apparaît comme un moyen d'éviter les doublons.

En Franche-Comté, c'est incontestablement à Belfort que l'intégration est la plus poussée : un seul cabinet pour les deux exécutifs, un seul directeur général des services et des directeurs généraux adjoints communs aux deux entités. Sont également communs les services fonctionnels : affaires juridiques, ressources humaines, finances, informatique. À un degré moindre d'intégration, la politique de la ville, la voirie, l'action culturelle sont des services partagés. La commune conserve en propre, outre l'état-civil, l'éducation et la petite enfance, alors que le développement économique, l'eau et l'assainissement, les déchets, la gestion des grands équipements d'intérêt communautaire (piscine, patinoire, écoles de musiques) dépendent totalement de la communauté d'agglomération, conformément aux compétences qui lui sont dévolues.

Entre la ville de Besançon et la CAGB, le transfert des transports publics, du Conservatoire National de Région ou de la collecte des ordures ménagères s'est traduit par le transfert des moyens, financiers et en personnels. Les services du plan, de la documentation, de la prospective, des financements européens ont été mutualisés. Mais il reste de grandes marges de manoeuvre en ce qui concerne la mutualisation des services du personnel, des finances ou même de l'administration générale.

La mutualisation des services entre les communes et les EPCI, sous réserve du respect de la réglementation européenne (Rey P.-S., 2007), apparaît ainsi comme une réponse adaptée aux critiques formulées quant à l'efficacité de l'intercommunalité. Au moment où la recomposition des territoires s'avère essentielle pour l'élaboration et la mise en oeuvre de politiques locales adaptées, la remise en cause de l'intercommunalité au motif d'inefficience budgétaire pourrait être interprétée comme une « recentralisation rampante » fort dommageable.

\section{BIBLIOGRAPHIE}

Actes du colloque, 2007, «Communes, communautés ; quelle mutualisation des services ? ", Palais du Luxembourg, Maires et Présidents de communautés de France, hors série, 27 mars 2007.

ADCF, 2007, Bilan des schémas d'orientation de l'intercommunalité, à partir de l'exploitation de 37 schémas, $28 \mathrm{p}$.

Bernard-Gélabert M.-C., 2004, « L'intercommunalité », 5e éd., LGDJ, IX, 113 p.

Chevailler J.-C., 2003, « Le Grand Besançon : du District à la Communauté d'Agglomération », Besançon, Images de Franche-Comté, n² 27, p. 10 à 13.

Chevailler J.-C., 2005, « Du SDAU au SCOT », 30 ans de planification du développement de l'agglomération bisontine, Besançon, Images de Franche-Comté, nº 32, p. 2 à 5.

Chevailler J.-C., 2007, « Le traitement des ordures ménagères dans la région de Besançon ", Images de Franche-Comté, n 36, p. 16 à 19. 
Dallier P., 2007, « Intercommunalité et fiscalité propre, bilan et perspectives », Observatoire de la décentralisation, Le Moniteur, cahier $\mathrm{n}^{\circ} 2,79 \mathrm{p}$.

Gilbert G., Guenfant A., Hespel H., 2001, Développement des territoires et financement des collectivités locales, in L'État et l'aménagement du territoire, Paris, DATAR, Documentation française, p. 193 à 218.

Intercommunalité, 2007, « L'après schémas ", Le répertoire administratif, n 1, janvier 2007, p. 6 à 11.

Marconis R., 2006, « Recompositions territoriales », La Documentation française, 64 p.

Mariton H., 2005, « Évolution de la fiscalité locale : le contribuable se rebiffe », Paris, Assemblée nationale, $476 \mathrm{p}$.

Massimi J.-M., 2002, Dictionnaire de l'intercommunalité, SEFI, 290 p.

Ministère du budget, des comptes publics et de la fonction publique, 2007, « Les comptes de l'intercommunalité et des établissements publics locaux en 2005 », La Documentation Française, $62 \mathrm{p}$.

Rey P.-S., 2007, « Mutualisation des services et mise en concurrence », France, La Gazette des Communes, $4 \mathrm{p}$.

Richard P., 2006, « Solidarité et Performance : les enjeux de la maîtrise des dépenses publiques locales », Observatoire des finances locales, $172 \mathrm{p}$.

Schémas d'orientation de l'intercommunalité, 2006, Préfectures du Doubs, du Jura, de la HauteSaône et du Territoire de Belfort.

\section{NOTES}

1. Source : Insee, population légale au RGP de 1999.

2. Loi n 66-1069 du 31 décembre 1966 relative aux communautés urbaines.

3. Loi d'orientation $\mathrm{n}^{\circ}$ 92-125 du 6 février 1992 relative à l'administration territoriale de la République.

4. Loi 99-586 du 12 juillet 1999 relative au renforcement et à la simplification de l'intercommunalité.

5. Loi du 4 février 1995 d'orientation pour l'aménagement et le développement du territoire et Loi du 25 juin 1999 d'orientation pour l'aménagement et le développement durable du territoire.

6. Loi du 13 décembre 2000 relative à la solidarité et renouvellement urbains.

\section{RÉSUMÉS}

La création des Établissements publics de coopération intercommunale et leur substitution progressive aux anciens syndicats favorisent la réalisation des conditions nécessaires à l'élaboration et à la mise en oeuvre des politiques locales d'équipement et d'aménagement du territoire. À l'issue de l'élaboration des Schémas départementaux d'orientation de 
l'intercommunalité, il est possible, en illustrant le propos par des exemples locaux qui révèlent des comportements départementaux sensiblement différents, de faire le bilan de ce vaste mouvement en Franche-Comté.

The creation of the "Etablissements publics de coopération intercommunale" and their gradual substitution to the former structures - known as "syndicats" - offer a much more satisfactory framework for defining and implementing local policies dealing with infrastructures and territorial development. As the "Schémas départementaux d'orientation de l'intercommunalité" have now been completed, it has become possible to assess this extensive evolution in FrancheComté, using various examples which reflect the differences from one district - "département" to the next.

Durch die Einrichtung der "Etablissements publics de coopération intercommunale" (öffentliche Einrichtung für kommunale Zusammenarbeit) wurden die ehemaligen "syndicats" (Kommunalverbände) schrittweise ersetzt. Dies begünstigte die Ausarbeitung und Umsetzung lokaler Infrastruktur - und Planungspolitiken. Nach Fertigstellung der "Schémas départementaux d'orientation de l'intercommunalité", welche sehr unterschiedliche Strategien der verschiedenen Departements erkennen lassen, ist es nun möglich, anhand lokaler Beispiele Bilanz für diesen bedeutenden Prozess in der Franche-Comté zu ziehen.

\section{INDEX}

Schlüsselwörter : Franche-Comté, Interkommunale Zusammenarbeit

Keywords : cooperation, Franche-Comté, intercommunality

Mots-clés : Franche-Comté, intercommunalité, jeu des acteurs

\section{AUTEURS}

\section{JEAN-CLAUDE CHEVAILLER}

Université de Franche-Comté - UMR 6049 TheMA - 45D av. de l'Observatoire - 25030 Besançon

Cedex - jean-claude.chevailler@univ-fcomte.fr

\section{PHILIPPE SIGNORET}

Université de Franche-Comté - UMR 6049 TheMA - 45D av. de l'observatoire - 25030 Besançon

Cedex - philippe.signoret@univ-fcomte.fr 